Journal of Pulmonary \& Respiratory Medicine: Volume 7, Issue 1

\section{Mustafa Özdal}

Gaziantep University, Physical Education and Sport Department, Gaziantep, Turkey

\section{Editorial Note}

It was realized lately that, urbanization took a high cost by increasing the death rate due to pollution and other hazardous toxicity towards human. Air-pollution is the prime cause underlying several pulmonary diseases, which is the fourth leading cause of death in USA and India tops among the countries suffering most from the pulmonary disorders [1]. Pulmonary diseases are a spectrum of disorders affecting the lungs, such as COPD, asthma, pulmonary hypertension, and lung cancer, infections like pneumonia tuberculosis, and influenza, and many other respiratory disorders and allergies. Plethora of research has been documented prophylactic ways for pulmonary disorders and offered adequate medicinal support to eradicate several lung deathly infections from the world like TB, Pneumonia etc. Taking the fact in mind the present Journal of 'Journal of Pulmonary \& Respiratory Medicine' showcased some interesting case studies like-lung carcinoma metastasizing to the appendix, cardio vocal syndrome manifesting as a symptom of pulmonary hypertension, assessment of the sociodemographic features of Tuberculosis patients, assessment of TB related knowledge among the patients, a follow-up study on multidrug resistant tuberculosis (MDRTB), markers delineating the severity of Progressive lung diseases, and assessment of allergic reactions to TrimethoprimSulfamethoxazole (TMP-SMX).

Lung adenocarcinoma is the most prominent cancer in the world with about 1.1 million deaths being attributed to it every year. About $85 \%-90 \%$ of the cases of lung adenocarcinoma are related to smoking. The main sites of metastasis of lung cancer are bone, liver, bone marrow, adrenal glands, kidney and central nervous system. Neto et al. [2], present a rare case of lung carcinoma metastasizing to the appendix. Appendicular carcinoid tumors are very are and account for approximately $0.52 \%$ of the appendicular diseases. The patient complained of abdominal pains and disturbance in bowel habits and was later diagnosed with lung cancer. Later on, the patient developed a distended abdomen with mild edema and lymphadenopathy of the lower limbs. CT scan of the pelvis revealed that the cancer had metastasized to the liver, bone, and heart. Immunohistochemistry revealed that the appendix was enlarged and that the adenocarcinoma had infiltrated the entire wall of the appendix, this was corrected by performing appendectomy.

In this issue, Bolaki et al. [3], present a unique case of idiopathic pulmonary arterial hypertension presenting with hoarseness of voice as the only symptom. The patient, a 40 year old woman complained of hoarseness of voice and did not exhibit any other symptom. Her daily routine was unhampered. Pulmonary function tests revealed the presence of pulmonary vascular disease and cardiopulmonary revealed cardiocirculatory origin. Heart catheterization revealed the presence of precapillary pulmonary hypertension, leading to a diagnosis of idiopathic pulmonary arterial hypertension. This is the first case report of cardiovocal syndrome (Ortner's syndrome) in a patient with IPH who exhibited no breathing problems and did not show any limitations with respect to daily activities.

The incidence of Tuberculosis is one of the key indicators of public health. Pulmonary tuberculosis is the predominant cause of morbidity and mortality in the third world. Unemployment, unhealthy living, and low level of education, may be the reason for the preponderance of TB in the lower socio economic strata. Raza et al. [4], conducted an observational study for assessing the clinical and socio-demographic characteristics of Tuberculosis patients in tertiary care. This study was conducted over a period of one year with 112 tuberculosis patients. The prominent symptoms observed in pulmonary tuberculosis patients were expectoration (96.5\%), fever (73.7\%), loss of appetite $(54.4 \%)$, and weight loss (80.7\%). The authors identified that the incidence of TB correlated positively with smoking as $50 \%$ of the patients were smokers. Only $8.9 \%$ of patients had an annual income in the excess of 2 lacs Taka. Further, the incidence of TB was identified to be gender dependent as the majority of the patients were female. The authors attributed it to the economic challenges such as illiteracy and lower income faced by women.

Extensively drug resistant TB (XDRTB) or Multidrug resistant tuberculosis (MDR-TB) poses the biggest challenge to public healthcare in recent times. In this issue, Rehman et al. [5], conducted a crosssectional study to assess TB related knowledge among the patients from the Pulmonary department of Owaisi Hospital and Research Center, Hyderabad. The authors found that majority of the patients (59\%) were unaware of the severe complications of TB. Compliance was also a major issue as a significant number of patients did not comply with the treatment and discontinued it in-between. The authors suggest that information regarding $\mathrm{TB}$ and other pulmonary disorders needs to be provided to common man, emphasis has to be given to the severe complications of the disease so that patients comply with the treatment in its entirety.

Periasamy and colleagues [6] has conducted a follow-up study on multidrug resistant tuberculosis (MDRTB) patients from 2011-2015. Univariate logistic regression was applied to the patient data. A total 63 MDRTB patients were followed and the outcomes reported as success or failure. Success included "compliance with entire treatment regimen" and "cured". Failure included death, untraceable, defaulted, and treatment failure. Of the total 63 cases, 33 registered as failed outcome and 30 were successful. The author found that diabetes, drug abuse, smoking and XDRTB suspects were the factors associated with failed outcome. The treatment outcome was independent of gender, age, HIV seropositivity, renal failure, and pre-treatment resistance profile.

Progressive lung diseases cause irreversible damage to pulmonary function. Therefore, markers delineating the severity of the disease are the need of the hour. Forced Expiration Volume in the first second $\left(\mathrm{FEV}_{1}\right)$ measured by spirometry, has traditionally been used for assessing pulmonary function. However, this technique is not sensitive enough to identify Cystic Fibrosis (CF) in its early stages. High

*Corresponding author: Mustafa Özdal, Gaziantep University, Physical Education and Sport Department, Gaziantep, Turkey, Tel: 905065150159; E-mail: ozdalm@hotmail.com

Received May 09, 2017; Accepted May 11, 2017; Published May 15, 2017

Citation: Özdal M (2017) Journal of Pulmonary \& Respiratory Medicine: Volume 7 Issue 3. J Pulm Respir Med 7: e136. doi: 10.4172/2161-105X.1000e136

Copyright: ( 2017 Özdal M. This is an open-access article distributed under the terms of the Creative Commons Attribution License, which permits unrestricted use, distribution, and reproduction in any medium, provided the original author and source are credited. 
Resolution Computed Tomography (HRCT) can identify disruptions in the lung structure, but the high level of radiation used for it; make it unsafe for multiple follow-ups in a short interval. Lung clearance index (LCI) is acknowledged to be more sensitive than spirometry. Multiple Breath Washout method (MBW) is a safe non-invasive test for assessing ventilation inhomogeneity, LCI being one of its parameters. Avramidou et al. [7], assessed the correlation of MBW with conventional lung function assays such as spirometry and structural read-outs such as HRCT. The authors observed that MBW parameters (LCI, LCI 5 , Sacin, Scond, $\mu_{1} / \mu_{0}$, and $\left.\mu_{2} / \mu_{0}\right)$ correlated significantly with spirometric and partial parameters of HRCT. However, it was observed that in mild conditions ventilation inhomogeneity indices associated better with CT data, while in mild- moderate conditions, MBW indices exhibited better correlation with spirometry.

Trimethoprim-Sulfamethoxazole (TMP-SMX) is a commonly prescribed antibiotic. One of the rare allergic reactions to TMP-SMX is severe systemic reaction which mimics sepsis or septic shock and is therefore a diagnostic challenge. Abbas et al. [8], present two case reports of diverse allergic reactions to TMP-SMX. The first patient developed a rash and fever post treatment with TMP-SMX. The infection report was negative and the patient improved with supportive care. The second patient presented systemic inflammatory response resembling septic shock. As with the first patient, the infection report was negative and the patient recovered with supportive care. The patient recovered completely with supportive care. Thus, one has to be careful while taking or prescribing TMP-SMX.

\section{References}

1. http://www.thehindu.com/news/national/andhra-pradesh/india-tops-world-inlung-disease-deaths/article7372468.ece

2. Neto CC, Kato SH, Pinto CAL, Santos GRD, Dettino ALA, et al. (2017) Metastatic Lung Adenocarcinoma to the Appendix: A Case Report. J Pulm Respir Med 6: 386.

3. Bolaki M, Fanaridis M, Pediaditis E, Mitrouska I, Georgopoulos D (2017) Ortner's Syndrome as First Clinical Presentation of Severe Pulmonar Arterial Hypertension. J Pulm Respir Med 6: 387.

4. Raza AKMM, Islam MR, Nahar M, Ahmed Z (2017) The Epidemiological Aspects of Tuberculosis Patients in a Tertiary Care Medical College Hospital of Bangladesh. J Pulm Respir Med 7: 389.

5. Rehman F, Raoof MA, Srinivasa Rao A, Hasan A, Qurram M (2017) Knowledge about Tuberculosis and its Complication among the Patients in a Teaching Hospital. J Pulm Respir Med 7: 390.

6. Periasamy A (2017) Predictors of Outcome in Drug Resistant Tuberculosis Patients. J Pulm Respir Med 7: 391.

7. Avramidou V, Hatziagorou E, Kampouras A, Georgopoulou V, Kirvasilis F, et al. (2017) Is Lung Clearance Index ( $\mathrm{CCl}$ ) Affected by the Severity of Lung Disease in CF? J Pulm Respir Med 7: 392

8. Abbas F, Patel M, Abbas K, Shah P, Gurel MN (2017) TrimethoprimSulfamethoxazole (TMP-SMX) Induced Severe Systemic Reaction. J Pulm Respir Med 6: 393 